\title{
Charge Carrier Transport in Non-Homogeneous MDMO-PPV Polymer
}

\author{
V. KaŽUkauskas*, M. Pranaitis And V. JanOnis \\ Department of Semiconductor Physics and Institute of Applied Research, Vilnius University \\ Saulètekio 9, bldg. 3, LT-10222 Vilnius, Lithuania
}

\begin{abstract}
By thermally stimulated currents we have investigated carrier transport and trapping in [poly-(2-methoxyl, 5-(3,77dimethyloctyloxy)] paraphenylenevinylene (MDMO-PPV). To assure selective excitation of the defect states the spectral width of the exciting light was varied from $1.77 \mathrm{eV}$ up to $3.1 \mathrm{eV}$. The thermally stimulated current curves were shown to be a superposition of carrier generation from trapping states and thermally stimulated mobility growth. The extrinsic excitation resulted in $0.16 \mathrm{eV}$ photoconductivity effective activation energy values, which decreased down to $0.05 \mathrm{eV}$ for the intrinsic excitation. The deeper states with activation energies of $0.28-0.3 \mathrm{eV}$ and $0.8-0.85 \mathrm{eV}$ were identified, too. The results are direct indication of distributed in energy trapping and transport states with the standard deviation of the density of states of about $0.015 \mathrm{eV}$.
\end{abstract}

PACS: 73.20.Hb, 73.50.Gr, 73.50.Pz, 73.61.Ph

\section{Introduction}

Poly(phenylen vinylen)s (PPVs) are distinguished by their excellent luminescent, mechanical properties and applicability for organic photovoltaic devices. MDMO-PPV [poly-(2-methoxyl, 5-(3,77dimethyloctyloxy)] para phenylenevinylene) is commonly used for the blends of the efficient organic solar cells. Nevertheless in PPVs fundamental charge transport problems still exist. The most of PPVs are good hole transport materials [1, 2]; meanwhile electrons are severely trapped. The determination of energetic distribution of trapping states is therefore of key importance. The distribution of the density of states (DOS) in PPV and its derivatives were demonstrated by indirect measurements in [3-5].

It is obvious that if traps are deep and situated far enough, distribution of their DOS over usually not more than $0.05 \mathrm{eV}$ does not affect significantly evaluation of the trap parameters, even if the simpler approach of localized traps is applied [6-8]. Though, distribution of the states close to the band edges is essential as they may start acting not only as traps but as transport states as well [9]. In this work we used thermally stimulated current measurements as direct photoelectrical method to prove distribution of trapping and transport states in MDMO-PPV.

\section{Experimental}

We have investigated $\approx 1 \mu \mathrm{m}$ layers of MDMO-PPV (Fig. 1). They were drop-casted on the indium-tin-oxide

* corresponding author; e-mail: vaidotas.kazukauskas@ff.vu.lt glass. The Al contact was deposited on the top. Carrier transport and trapping were investigated by the thermally stimulated current (TSC) spectroscopy as described in [8]. The samples at $77 \mathrm{~K}$ were excited by light with different spectral ranges to assure selective excitation of the defect states. As bandgap of MDMO-PPV is about $2.2 \mathrm{eV}$, excitation below and above band-gap by light passed through the long-pass colour filters with cut-on energies from $1.77 \mathrm{eV}$ up to $3.1 \mathrm{eV}$ was used. The curves were fitted by taking into account thermal carrier generation from traps as described in [8-10] and their mobility variation according to the Gaussian disorder model (GDM) [11].

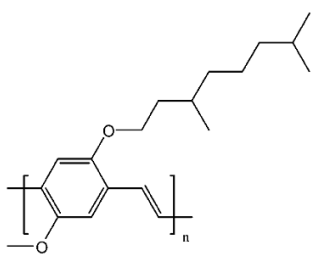

Fig. 1. Chemical structure of MDMO-PPV.

\section{Results and discussion}

In Fig. 2 the TSC curves are presented for different cut-off energies of the long-pass filters. Upon widening of the light spectra, the TSCs grow up to the saturation which is achieved when quantum energy exceeds the band gap. This growth is accompanied by the reduction of the effective activation energy value of the current maximum appearing in the lowest curve at about $240 \mathrm{~K}$. Apart from 
that a slight shift of the maximum to the lower temperatures takes place. Approaching the room temperature a steeper current increase starts with the thermal activation energy value of about $0.28-0.3 \mathrm{eV}$ that is caused by carrier thermal generation from deeper trapping states. Close to the room temperature deeper states with activation energies of $0.8-0.85 \mathrm{eV}$ become active. The curves were fitted (Fig. 3) by taking into account carrier thermal generation from trapping states and their mobility variation with temperature as described in [8, 9, 11]. At low temperatures TSCs are essentially influenced by mobility growth, appearing at the background of thermally stimulated current maxima due to the thermal generation of carriers. The latter mechanism is dependent on excitation conditions by light (Fig. 2) as will be discussed below.

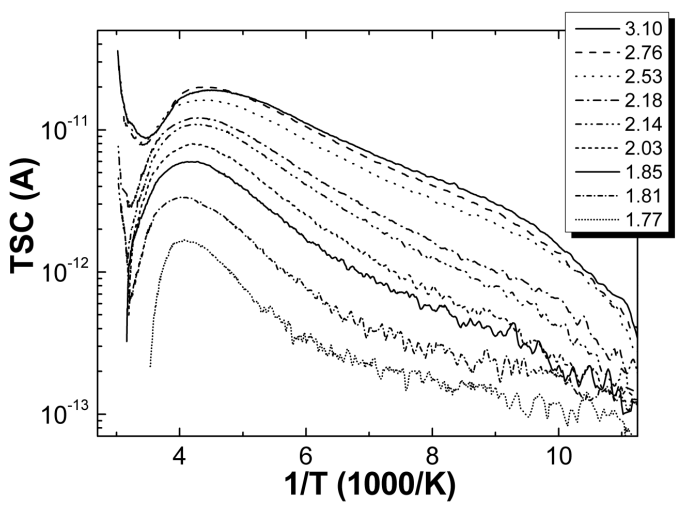

Fig. 2. TSC curves depending on the cut-off energies of the long-pass colour filters.

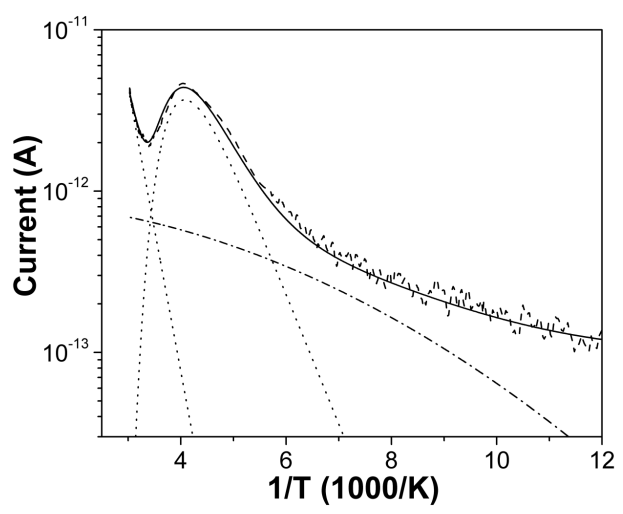

Fig. 3. Fitting of the experimental TSC, measured after the light excitation with the cut-on energy $1.77 \mathrm{eV}$. Dashed line shows experimental data, solid curve is fitting results, dotted lines represent thermal generation of carriers from trapping states, and dashed-dotted curves show mobility variation according to GDM.

By fitting the mobility parameters were kept constant, just the temperature was varied. The following GDM [11] parameters were used: $\Sigma \approx 1, \sigma \approx 0.025 \mathrm{eV}$, $\mu_{\infty} \approx 8 \times 10^{-4} \mathrm{~cm}^{2} /(\mathrm{Vs})$, and $C \approx 3 \times 10^{-4}(\mathrm{~cm} / \mathrm{V})^{0.5}$.
Similar values were obtained in P3HT $[12,13]$ and in MEH-PPV [8]. Characteristically the higher spatial disorder parameter $\Sigma=4.5$ was evaluated in P3HT:PCBM solar cells [9], evidencing their greater spatial disorder because of the bulk heterojunction structure. On the other hand, energetical disorder parameter $\sigma \approx 0.06-0.07 \mathrm{eV}$ was higher in P3HT and P3OT [12, 13], but in their structures with PCBM it was comparable to that in our samples, i.e., 0.028-0.045, indicating more favourable transport conditions. Moreover very similar values of $\mu_{\infty}$ indicate that charge transport in solar cell structures is primarily governed by polymer, not by PCBM, having higher mobilities.

We will focus on the trap parameters, obtained by fitting, as in Fig. 3. In Fig. 4 density of the filled trapping states $n$ is presented depending on the exciting light spectral edge. If the light quanta energy is below the band gap only a small fraction of the traps becomes filled. As the quanta energy exceeds the band gap, trap filling tends to saturate at $(5-6) \times 10^{15} \mathrm{~cm}^{-3}$. This value coincides with the data of MDMO-PPV [14] and MEH-PPV [8]. The higher densities of traps at the interfaces of the Schottky junction were reported in [4]. The trap density increases in bulk heterojunction devices as well, most probably also at the interfaces $[9,15]$. It is seen in Fig. 4 that a big number of trapping states exists close to the band gap, which can be excited by the extrinsic light. The lower quantum energy of the exciting light the deeper trapping centres are excited. Therefore their thermal activation energy will be higher. On the other hand, by increasing quantum energy trapping states closer to band gap edge will be excited, therefore obtained effective activation energy will decrease, as it is confirmed by Fig. 5 .

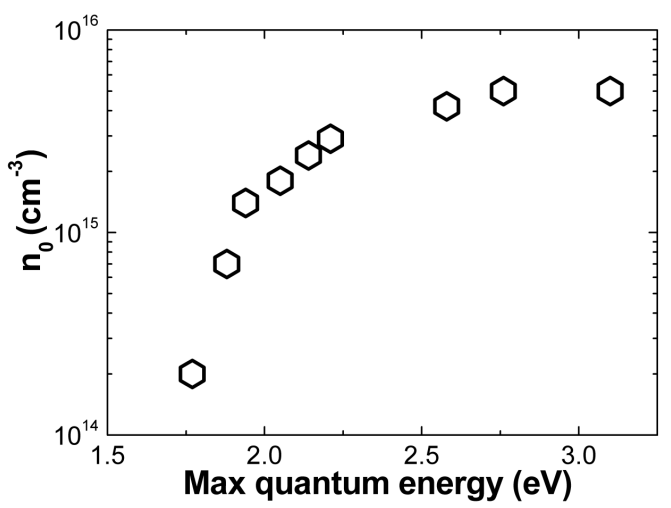

Fig. 4. Density of the filled trapping states $n_{0}$ depending on the exciting light spectral edge.

In Fig. 5 initial trap filling values are shown depending on their effective activation energy. In the same figure the Gaussian distribution is plotted

$$
n_{0}\left(E, E_{0}, \sigma^{2}\right)=\frac{N_{0}}{\sqrt{2 \pi \sigma^{2}}} \exp \left(-\frac{\left(E-E_{0}\right)^{2}}{2 \sigma^{2}}\right) .
$$

Here $E$ is the activation energy value, $E_{0}$ is its median, and $\sigma^{2}$ is the variance. The FWHM of the curve is given 


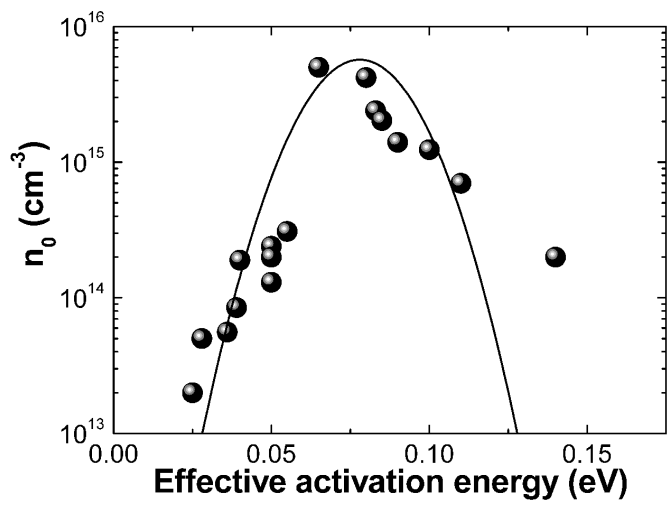

Fig. 5. Initial trap filling vs. their effective activation energy. The solid curve represents Gaussian distribution with $\Gamma=0.035 \mathrm{eV}$ and $E_{0}=0.078 \mathrm{eV}$.

by $\Gamma=2 \sqrt{2 \ln \sigma}$. The experimental data qualitatively follow Gaussian distribution with the following parameters: $\sigma=0.015 \mathrm{eV}, \Gamma=0.035 \mathrm{eV}$ and $E=0.078 \mathrm{eV}$. The FWHM of the trapping state distribution coincides with the energetic disorder parameter in GDM. This indicates that the relatively shallow trapping states can be caused by the band edge tails, and therefore they might be acting as transport states, if carriers are energized enough to overcome these barriers.

Moreover, existence of deeper traps is seen in Figs. 2, 3. Their thermal activation energies range from 0.28 to $0.3 \mathrm{eV}$ and from 0.8 to $0.85 \mathrm{eV}$. Similar values were also found in MEH-PPV [8], indicating similar nature of the trapping states.

In summary, our results are direct indication by the photo-thermo-electrical methods of the distributed in energy trapping and transport states with approximate standard deviation of the density of states of $0.015 \mathrm{eV}$.

\section{References}

[1] P.W.M. Blom, M.J.M. de Jong, J.J.M. Vleggaar, Appl. Phys. Lett. 68, 3308 (1996).

[2] H. Antoniadis, M.A. Abkowitz, B.R. Hsieh, Appl. Phys. Lett. 65, 2030 (1994).

[3] J. Nelson, Phys. Rev. B 67, 155209 (2003).

[4] A.J. Campbell, D.D.C. Bradley, E. Werner, W. Bruetting, Org. Electron. 1, 21 (2000).

[5] L. Gorisa, A. Poruba, L. Hod'ákova, M. Vaněček, K. Haenen, M. Nesládek, P. Wagner, D. Vanderzande, L. De Schepper, J.V. Mancab, Appl. Phys. Lett. 88, 052113 (2006).

[6] J. Steiger, R. Schmechel, H. Von Seggern, Synth. Met. 129, 1 (2002).

[7] T.P. Nguyen, P. Le Rendu, P. Leveque, J. Ip, O. Gaudin, R.B. Jackman, Org. Electron. 5, 53 (2004).

[8] V. Kažukauskas, Semicond. Sci. Technol. 19, 1373 (2004).

[9] V. Kažukauskas, A. Arlauskas, M. Pranaitis, M. Glatthaar, A. Hinsch, J. Nanosci. Nanotechnol. 10, 1376 (2010).

[10] J.G. Simmons, G.W. Taylor, Phys. Rev. B 5, 1619 (1972).

[11] H. Baessler, Phys. Status Solidi 175, 15 (1993).

[12] A.J. Mozer, N.S. Sariciftci, A. Pivrikas, R. Österbacka, G. Juška, L. Brassat, H. Bässler, Phys. Rev. B 71, 035214 (2005)

[13] V. Kažukauskas, M. Pranaitis, V. Čyras, L. Sicot, F. Kajzar, Thin Solid Films 516, 8988 (2008).

[14] G. Dennler, A.J. Mozer, G. Juška, A. Pivrikas, R. Osterbacka, A. Fuchsbauer, N.S. Sariciftci, Org. Electron. 7, 229 (2006).

[15] M.M. Mandoc, W. Veurman, L.J.A. Koster, M.M. Koetse, J. Sweelssen, B. de Boer, P.W.M. Blom, J. Appl. Phys. 101, 104512 (2007). 